\title{
Effects of phased joint intervention on Rho/ROCK expression levels in patients with portal hypertension
}

\author{
MIN SHI, JUE WEI, WEN-YING MENG, NA WANG, TING WANG and YU-GANG WANG \\ Department of Gastroenterology, Shanghai Tongren Hospital, \\ Affiliated to Shanghai Jiao Tong University School of Medicine, Shanghai 200336, P.R. China
}

Received March 29, 2015; Accepted May 11, 2016

DOI: $10.3892 /$ etm.2016.3454

\begin{abstract}
The current study investigated the effects of phased joint intervention on clinical efficacy and Rho/Rho-associated coil protein kinase (ROCK) expression in patients with portal hypertension complicated by esophageal variceal bleeding (EVB) and hypersplenism. Patients with portal hypertension $(n=53)$ caused by liver cirrhosis complicated by EVB and hypersplenism treated with phased joint intervention were assessed, and portal hemodynamics, blood, liver function, complications, and rebleeding incidence were analyzed. Reverse transcription-quantitative polymerase chain reaction was used to measure Rho, ROCK1 and ROCK2 mRNA expression levels in peripheral blood mononuclear cells prior to and following phased joint intervention, and western blotting was employed to determine the protein expression levels of Rho, ROCK1, ROCK2, phosphorylated (p) myosin phosphatase target subunit 1 (MYPT1) and total-MYPT1. All patients underwent an emergency assessment of hemostasis with a $100 \%$ success rate. Varicose veins were alleviated, and portal hemodynamics and liver function improved following intervention. Furthermore, preoperative and postoperative expression levels of Rho, ROCK1 and ROCK2 mRNA were higher compared with the control group. Notably, the mRNA expression levels of Rho, ROCK1 and ROCK2 in the postoperative group were significantly lower when compared with the preoperative group. Protein expression levels of Rho, ROCK1, ROCK2 and pMYPT1 in the postoperative group were lower, as compared with the preoperative group. Concentration levels
\end{abstract}

Correspondence to: Professor Yu-Gang Wang, Department of Gastroenterology, Shanghai Tongren Hospital, Affiliated to Shanghai Jiao Tong University School of Medicine, 1111 Xianxia Street, Shanghai 200336, P.R. China

E-mail:wangyugang2013@yeah.net

Abbreviations: PTVE, percutaneous transhepatic variceal embolization; EVB, esophageal variceal bleeding; PSE, partial splenic embolization; HSCs, hepatic stellate cells; DSA, digital subtraction angiography; ROCK, Rho-associated coil protein kinase; CTGF, Connective tissue growth factor.

Key words: esophageal varices, embolization, cirrhosis, Rho, rock of transforming growth factor- $\beta 1$, connective tissue growth factor and platelet-derived growth factor in peripheral blood were significantly reduced following phased joint intervention. Therefore, the present findings demonstrated that phased joint intervention is able to effectively treat EVB and hypersplenism, and improve liver function. The efficacy of phased joint intervention may be associated with its role in the regulation of the Rho-ROCK signaling pathway.

\section{Introduction}

Cirrhosis is associated with portal hypertension. Esophageal variceal bleeding (EVB) is is one of the most common and dangerous complications of cirrhosis associated with portal hypertension (1). The majority of patients suffer from acute onset of the disease, which is accompanied by high mortality rates due to the large amount of bleeding that is difficult to stop (2). Approximately $50 \%$ of patients with cirrhosis are diagnosed with esophageal varices at the outset, and those with Child-Pugh Class B or C liver function are susceptible to a higher incidence of esophageal varices (3), with $7 \%$ developing esophageal varices each year $(4,5)$. The rate of initial hemorrhage is $\sim 12 \%$ in the first year (6), and the recurrence rate of hemorrhage after 1 year is $\sim 60 \%$ (7). Mortality rates within 6 weeks of bleeding remain high at up to $20 \%$ (8).

Since 1974, percutaneous transhepatic variceal embolization (PTVE) has been used for the effective treatment of EVB (9). However, its clinical application is limited to patients with a high rate of rebleeding (10-12). Transcatheter splenic arterial embolization can decrease portal vein blood flow and pressure, and improve the symptoms of hypersplenism; however, its benefits are limited as $73 \%$ of patients experience severe complications if the embolic volume is $>70 \%$ of the vein (13). Since 2006, our group has used the improved phased joint intervention $[\mathrm{PTVE}+$ staged partial splenic embolization (PSE)] to treat portal hypertension complicated by EVB and hypersplenism. This avoids the weaknesses of other therapeutic methods, thus achieving satisfactory clinical efficacy. In the current study the clinical applications of phased joint intervention were explored by reviewing the case data. However, the mechanism of action of this therapeutic method has yet to be elucidated.

The central features of the occurrence and progression of liver cirrhosis are the activation and proliferation of 
hepatic stellate cells (HSCs). In vitro studies have revealed that extracellular pressure promotes the proliferation of HSCs and increase the expression levels of associated cytokines and other extracellular matrix (ECM) components, such as collagen $(14,15)$. Phased joint intervention improves liver hemodynamics, which has a major impact on liver restructuring and alters the mechanical environment of the HSCs (16). Rho small G proteins have an important role in mechanical transduction. A previous study indicated that the aforementioned proteins are able to regulate various biological activities of HSCs by activating crucial downstream effectors, including Rho-associated coil protein kinase (ROCK) (17). The present study further investigated the protein and mRNA expression levels of Rho and ROCK1/ROCK2 prior to and following interventional embolization, and explored the possible mechanisms underlying the effect of embolization on the progression of liver cirrhosis.

\section{Materials and methods}

Patients and ethics statement. The present retrospective study involved 53 cases of liver cirrhosis complicated by portal hypertension, EVB and hypersplenism treated in Shanghai Tongren Hospital (Shanghai, China) from October 2006 to December 2011. Portal hypertension was predominantly due to hepatitis (35 cases), autoimmunity (10 cases) or alcoholic cirrhosis (8 cases), with phased joint intervention (PTVE + phased PSE) selected as the interventional therapy. The current study included 37 men and 16 women, with an average age of $47.92 \pm 8.0$ years (range, $44-71$ years). The liver function of the enrolled patients was classified as Child-Pugh class $A(n=15)$, class $B(n=22)$ or class $C(n=16)$. All 53 patients had a history of $\geq 1$ bleeding episodes, and received emergency interventional treatment. Ethical approval was granted by the Shanghai Changning District Central Hospital Ethics Committee. All patients agreed to accept the intervention, and they (or their authorized family members) provided written informed consent. Inclusion and exclusion criteria were performed as previously described by Wang et al (16).

Materials. Primers of RhoA, ROCK1/ROCK2 were designed by the current group and synthesized by Shanghai Generay Biotech Co., Ltd. (Shanghai, China) (Table I). Rabbit anti-ROCK1 (C8F7) monoclonal antibody (mAb; cat. no. 4035), rabbit anti-ROCK2 (D1B1) mAb (cat. no. 9029), rabbit anti-RhoA (67B9) mAb (cat. no. 2117), rabbit anti-myosin phosphatase target subunit 1 (MYPT1) (D6C1) mAb (cat. no. 8574) and rabbit anti-phosphorylated (p)-MYPT1 (Thr853) polyclonal antibody (cat. no. 4563) were all purchased from (Cell Signaling Technology, Danvers, MA, USA). Mouse anti- $\beta$-actin mAb (cat. no. A5316), goat anti-rabbit IgG (whole molecule)-peroxidase antibody (cat. no. A0545) and goat anti-mouse IgG (Fc-specific)-peroxidase antibody (cat. no. A0168) were purchased from Sigma-Aldrich, (St. Louis, MO, USA). SYBR Green Real-Time PCR Master Mix (Toyobo Co., Ltd., Osaka, Japan); Pierce BCA Protein Assay kit (Thermo Fisher Scientific, Inc., Waltham, MA, USA); Amersham ECL Plus Western Blotting Detection System (GE Healthcare Life Sciences, Chalfont, UK); ELISA kits (cat. nos. CB5683, OK0109 and OK0155; Assay
Biotechnology Company Inc, Sunnyvale, CA, USA), GE3100 flat C-DSA system; and a GE730 Color Doppler ultrasound diagnostic apparatus, all GE Healthcare Life Sciences).

Phased joint embolization. For PTVE + PSE, the PSE embolization range was controlled between 30 and 40\%. PSE was conducted again three months later with an embolization range controlled between 30 and $40 \%$.

PTVE. Using a 21 gauge needle (Cook Medical, Bloomington, IN, USA), the right portal vein was percutaneously and transhepatically punctured with following routine sterilization and positioning of the liver. Following successful puncture, a guide wire was inserted into the superior mesenteric vein along the needle, and a $5 \mathrm{~F}$ vascular sheath (Cook Medical) was subsequently inserted using the guide wire. The contrast agent (iohexol) was injected to perform splenic and portal vein digital subtraction angiography (DSA). To visualize the esophageal varices, the catheter tip was superselectively inserted into the gastric coronary vein. For distal embolization of the gastric coronary vein, 5-30 ml absolute ethanol was injected according to the radiography situation of DSA. In cases with particularly slow blood flow, a spring ring of the appropriate specification (Cook Medical) was utlilized for main vein thrombosis. In the event that the angiographic image revealed complete occlusion of the gastric coronary vein, short gastric vein thrombosis was treated according to the same method. In cases with particularly rapid blood flow, a steel ring (diameter, $5-10 \mathrm{~mm}$ ) or gelatin sponge particles were used for embolization to reduce the speed of the blood flow in the thickened varicose branch. Absolute ethanol was gradually injected until the varicose vein was no longer visible to the naked eye. In order to determine the embolization effect, DSA was conducted for a second time. During this secondary DSA, the catheter tip was once again inserted into the splenic vein to confirm whether there were any varicose veins. If varicose veins were detected, the same method was applied, as previously described, for complete embolization. Following treatment, the catheter sheath was carefully removed from the right portal vein to reduce the risk of bleeding. In the event of bleeding, a gelatin sponge was used to block the needle tract with a pressure dressing to ensure no further bleeding for $48 \mathrm{~h}$.

PSE. The catheter was inserted using Seldinger's technique via the femoral artery to reach the splenic hilum, and its medium and lower branches via the proximal splenic artery. The splenic vessels and blood flow were assessed by DSA, and gelatin sponge particles were injected into the splenic artery and its branches for embolization. Splenic arteriography was conducted, and the puncture point was subsequently subjected to pressure dressing once the catheter had been removed.

Postoperative treatment. Vital signs and abdominal conditions were closely observed. Patients receiving a pressure dressing and compression hemostasis were required to have undergone $24 \mathrm{~h}$ bed rest and to have taken preventive measures, including ceftazidime injection ( 2 g/day; Shandong Luoxin Group Pharmaceutical, Co., Ltd., Shandong, China), in order to prevent infection and other complications. 
Assessment of efficacy. Patients underwent re-examination for routine blood tests, liver function, gastroscopy, color Doppler ultrasound and computed tomography. Follow-up at 6 months included routine blood tests, and assessment of liver function, complications and rebleeding. In the event of gastrointestinal bleeding, patients visited the doctor immediately. Postoperative portal vein hemodynamics were evaluated by abdominal ultrasound. Postoperative indicators of liver function included alanine transaminase (ALT), albumin, total bilirubin (TBIL) and prothrombin time (PT-INR); whereas the indicators for hypersplenism improvement included white blood cell (WBC) and platelet counts. The aforementioned indicators were observed prior to intervention and 1, 4 (1 month after phased PSE) and 6 months after surgery. Serum levels of transforming growth factor (TGF)- $\beta 1$, connective tissue growth factor (CTGF) and platelet-derived growth factor (PDGF) were analyzed using ELISA kits.

Isolation, cryopreservation and resuscitation of peripheral blood mononuclear cells (PBMCs). Prior to surgery and 1, 4 and 6 months after intervention, $5 \mathrm{ml}$ fresh blood was collected from the patients in an EDTA anticoagulant tube, with 32 healthy samples used as controls. The plasma was stored at $-70^{\circ} \mathrm{C}$, and the remaining cells, resuspended in phosphate buffered saline, were carefully overlayed onto Ficoll and centrifuged at $800 \mathrm{x}$ g for $15 \mathrm{~min}$ at room temperature. Finally, purified PBMCs were nitrogen-frozen in RPMI 1640 supplemented with $10 \%$ DMSO and $10 \%$ fetal serum.

Reverse transcription-quantitative polymerase chain reaction $(R T-q P C R)$. Total RNA was isolated from PBMCs using TRIzol reagent (Invitrogen; Thermo Fisher Scientific, Inc.), according to the manufacturer's protocol, and treated with DNase (Promega Corporation, Madison, WI, USA). The purity and concentration of the RNA were determined by the absorbance at $260 \mathrm{~nm}$ and $280 \mathrm{~nm}$ using a spectrophotometer. RNA $(2 \mu \mathrm{g})$ was subsequently reverse transcribed into cDNA using PrimeScript ${ }^{\circledR}$ 1st Strand cDNA Synthesis kit (Takara Biotechnology Co., Ltd., Dalian, China). qPCR was performed using the SYBR Green Real-Time PCR Master Mix in an ABI PRISM 7900HT Sequence Detector (Thermo Fisher Scientific, Inc.). The PCR conditions included an initial denaturation at $94^{\circ} \mathrm{C}$ for $5 \mathrm{~min}$, followed by 40 cycles at $94^{\circ} \mathrm{C}$ for $30 \mathrm{sec}$, $61^{\circ} \mathrm{C}$ for $45 \mathrm{sec}$ and $72^{\circ} \mathrm{C}$ for $30 \mathrm{sec}$, and a final extension step at $72^{\circ} \mathrm{C}$ for $5 \mathrm{~min}$. Relative mRNA expression levels were calculated using the $2^{-\Delta \Delta \mathrm{Cq}}$ method (18), with normalization to $\beta$-actin. The primer sequences are displayed in Table I. All experiments were at least duplicated.

Western blotting. Total protein was extracted from PBMCs using SDS cell lysis buffer (Beyotime Institute of Biotechnology, Haimen, China) containing $1 \mathrm{mM}$ phenylmethylsulfonyl fluoride. Protein concentrations were measured using the Pierce BCA Protein Assay kit. Equal quantities of proteins $(100 \mu \mathrm{g})$ were separated by $10 \%$ SDS-PAGE and subsequently transferred onto nitrocellulose membranes. Membranes were blocked with $5 \%$ milk in Tris-buffered saline supplemented with Tween-20 (TBST) for $1 \mathrm{~h}$ at room temperature, then incubated with rabbit anti-ROCK1 (C8F7) mAb, anti-ROCK2 (D1B1) mAb, anti-RhoA (67B9) mAb, anti-MYPT1 (D6C1)
Table I. Primer probe sequences used in reverse transcription-quantitative polymerase chain reaction.

\begin{tabular}{lll}
\hline mRNA & \multicolumn{1}{c}{ Sequence (5'-3') } & $\begin{array}{c}\text { Amplicon } \\
\text { size (bp) }\end{array}$ \\
\hline RhoA & $\begin{array}{l}\text { F: GGAAAGCAGGTAGAGTTGGCT } \\
\text { R: GGCTGTCGATGGAAAAACACAT }\end{array}$ & 118 \\
ROCK1 & $\begin{array}{l}\text { F: AAGTGAGGTTAGGGCGAAATG } \\
\text { R: AAGGTAGTTGATTGCCAACGAA }\end{array}$ & \\
& F: TTGCTCTGGATGCAATACACTC & 223 \\
ROCK2 & R: TCTCGCCCATAGAAACCATCA & \\
F-actin & F: TGGAGAAAATCTGGCACCA & 189 \\
& R: CAGGCGTACAGGGATAGCAC & \\
\hline
\end{tabular}

F, foward; R, reverse; ROCK, Rho/Rho-associated coil protein kinase.

mAb and anti-p-MYPT1 (Thr853) polyclonal antibody, and mouse anti- $\beta$-actin $\mathrm{mAb}$ (all 1:1,000), at $4^{\circ} \mathrm{C}$ overnight. After washing three times for $10 \mathrm{~min}$ each with TBST, the membranes were incubated with peroxidase-conjugated goat anti-rabbit and goat anti-mouse secondary antibodies (1:5,000) for $1 \mathrm{~h}$ at room temperature. Membranes were visualized using the Amersham ECL Plus Western Blotting Detection System and analyzed using ImageJ $1.48 \mathrm{v}$ software (https://imagej.nih. gov/ij/). Relative protein expression levels were normalized to $\beta$-actin. All experiments were repeated in triplicate.

Statistical analysis. Data were analyzed using SPSS statistical software (version 13.0; SPSS, Inc., Chicago, IL, USA), and expressed as mean \pm standard deviation. Multiple groups were analyzed with one-way analysis of variance; pairwise comparison was conducted via a least significant difference t-test, and different groups were compared using a t-test. $\mathrm{P}<0.05$ was considered to indicate a statistically significant difference.

\section{Results}

Success rate of surgery. All 53 patients successfully received the joint intervention with emergency hemostasis (Fig. 1). Bleeding ceased immediately following surgery with an emergency hemostatic rate of $100 \%$. Varicose veins were significantly reduced in portal vein angiography after PTVE intervention, and partial spleen embolization occurred after PSE intervention (16). During the 6 months of follow-up, all patients survived and none reported rebleeding. Following phased joint interventional embolization, 32 patients exhibited various degrees of fever with body temperatures ranging from $37.5-39.4^{\circ} \mathrm{C}$, which was alleviated by symptomatic treatment. Postoperative pain was experienced by 29 patients, and was also relieved by symptomatic treatment. No serious complications occurred.

Quantitative determination of Rho, ROCK1 and ROCK2 expression levels by $R T-q P C R$. RT-qPCR analysis demonstrated that the mRNA expression levels of Rho, ROCK1 and ROCK 2 were significantly higher preoperatively and postoperatively, as compared with the normal control group $(\mathrm{P}<0.01)$. 


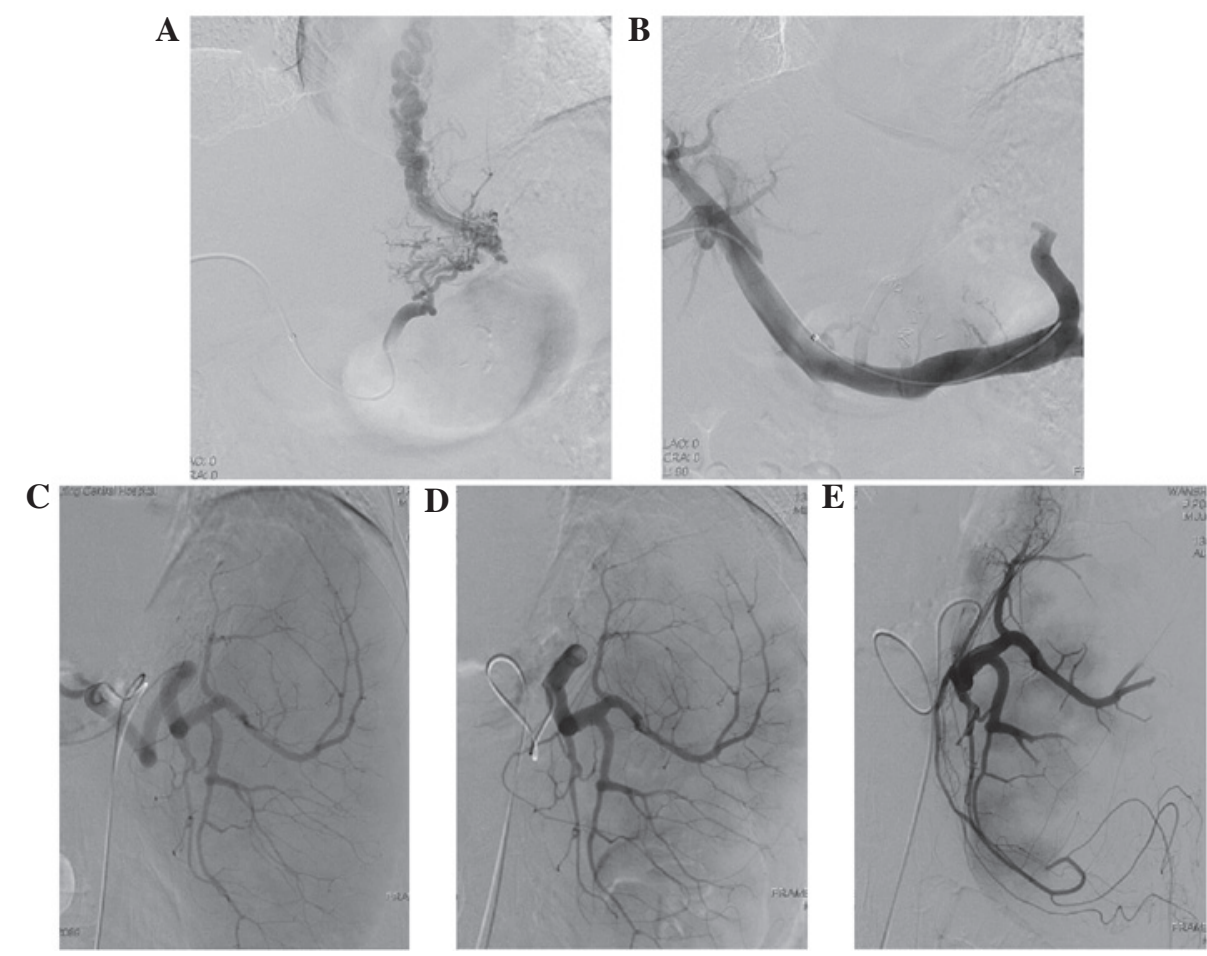

Figure 1. Changes in esophageal varices prior to and after treatment, demonstrated by digital subtraction angiography (DSA) . (A) Varicose vein as revealed by portal vein angiography prior to intervention. (B) Percutaneous transhepatic variceal embolization varicose vein occlusion following intervention. (C) Enlarged spleen demonstrated by DSA prior to partial splenic embolization (PSE) intervention. (D) Partial spleen embolization after PSE intervention. (E) Enlarged spleen embolization after repeat PSE intervention 3 months later.

However, the mRNA expression levels of Rho, ROCK1 and ROCK2 were significantly lower in the postoperative group, as compared with the preoperative group $(\mathrm{P}<0.01 ;$ Fig. 2$)$.

Determination of Rho, ROCK1, ROCK2, pMYPT1 and tMYPT1 protein expression levels by western blotting. Western blotting indicated that the protein expression levels of Rho, ROCK1 and ROCK2 were significantly higher in the preoperative and postoperative groups, as compared with the normal control group $(\mathrm{P}<0.05)$. However, the protein expression levels of Rho, ROCK1 and ROCK 2 were significantly decreased in the postoperative group, as compared with the preoperative group $(\mathrm{P}<0.05)$. The changes in protein expression levels were consistent with those of mRNA expression levels. Postoperative pMYPT1 protein expression levels were significantly reduced, as compared with preoperative levels $(\mathrm{P}<0.05)$, although there was no obvious change in tMYPT1 protein expression levels (Fig. 3).

Determination of TGF- $\beta 1, C T G F$ and PDGF in peripheral blood by ELISA. ELISA indicated that TGF- $\beta 1$, CTGF and PDGF concentrations in peripheral blood following phased joint intervention were significantly lower than the preoperative values ( $\mathrm{P}<0.01$; Fig. 4).

\section{Discussion}

PTVE predominantly embolizes the gastric coronary vein and short gastric vein to block the blood flow of varicose veins and stop bleeding, which is a proven method to treat portal hypertension and EVB $(9,19)$. This method may increase portal vein

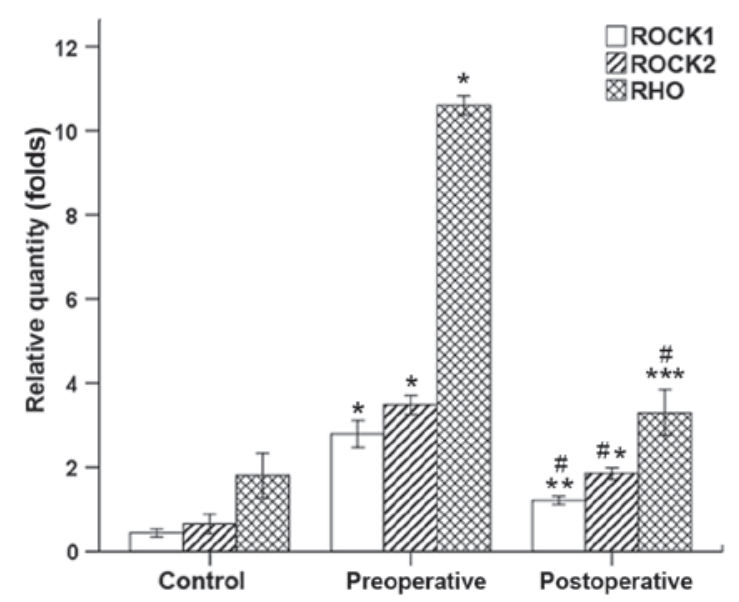

Figure 2. Rho, ROCK1 and ROCK2 expression levels before and after treatment, as determined by reverse transcription-quantitative polymerase chain reaction. mRNA expression levels of Rho, ROCK1 and ROCK2 were higher compared with the normal control group Prior to and after intervention (Rho: preoperative, $10.59 \pm 0.19$ vs. $1.80 \pm 0.46$; postoperative, $3.29 \pm 0.47$ vs. $1.80 \pm 0.46$; ROCK1: preoperative, $2.79 \pm 0.27$ vs. $0.44 \pm 0.08$; postoperative, $1.21 \pm 0.09$ vs. $0.44 \pm 0.08$; and ROCK 2 : preoperative, $3.48 \pm 0.19$ vs. $0.65 \pm 0.20$; postoperative, $1.84 \pm 0.11$ vs. $0.65 \pm 0.20$ ). mRNA expression levels of Rho, ROCK 1 and ROCK 2 in the postoperative group were significantly lower compared with those of the preoperative group (Rho: $3.29 \pm 0.47$ vs. $10.59 \pm 0.19$; ROCK1: $1.21 \pm 0.09$ vs. $2.79 \pm 0.27$; and ROCK2: $1.84 \pm 0.11$ vs. $3.48 \pm 0.19$ ). ${ }^{*} \mathrm{P}<0.001,{ }^{* *} \mathrm{P}=0.002$ and ${ }^{* * *} \mathrm{P}=0.004$ vs. the control group; ${ }^{*} \mathrm{P}<0.001$ vs. the preoperative group. ROCK, Rho/Rho-associated coil protein kinase.

pressure and bleeding rate, therefore, its clinical applications are limited. PSE intervention may decrease splenic blood flow and return flow of the splenic vein, release the high post-hepatic pressure, reduce portal blood flow and pressure, lower the 
A

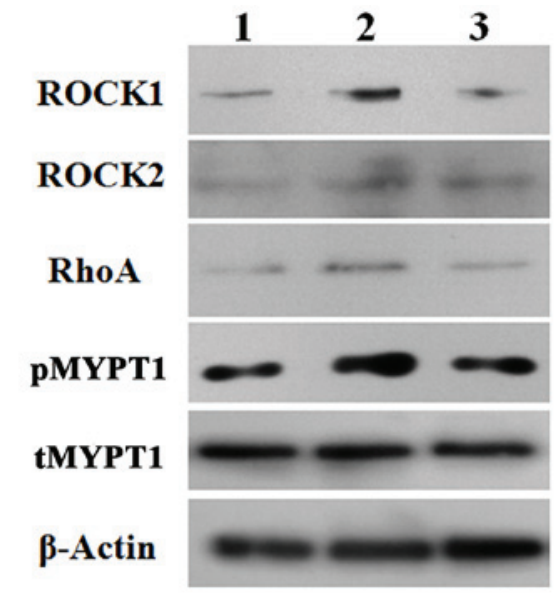

B

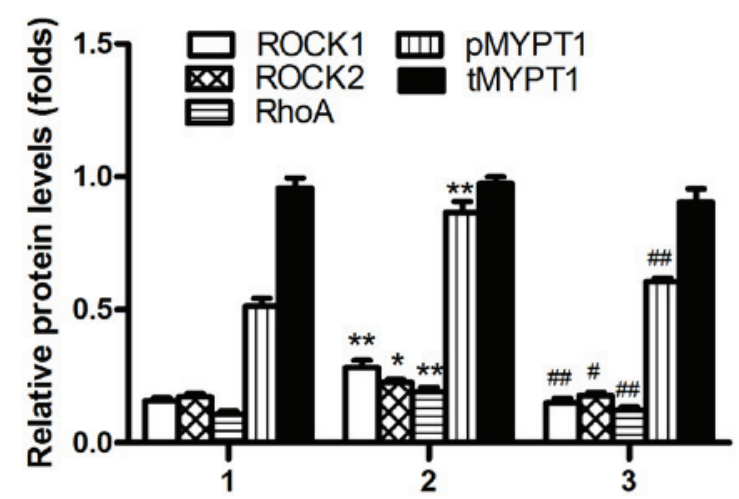

Figure 3. Protein expression levels of Rho, ROCK1 and ROCK2 prior to and after treatment, as determined by (A) western blotting and (B) densitometry. Protein expression levels were normalized to $\beta$-actin. 1, normal control group; 2, preoperative group; 3 , postoperative group. Data are presented as the mean \pm standard deviation. ${ }^{*} \mathrm{P}<0.05,{ }^{* *} \mathrm{P}<0.01$ vs. the control group; ${ }^{\#} \mathrm{P}<0.05,{ }^{\# \#} \mathrm{P}<0.01$ vs. the preoperative group. ROCK, Rho/Rho-associated coil protein kinase; $\mathrm{p}$ phosphorylated; t, total; MYPT1, myosin phosphatase target subunit 1 .

recurrence of esophageal and gastric variceal bleeding, and improve symptoms of hypersplenism (20).

Previous studies have suggested that it is difficult to return blood flow to normal levels if the splenic embolization area is $<40 \%$, and serious adverse reactions may occur if the embolization area is $>80 \%$ (21). Pålsson et al (22) reported that the embolization area is associated with an increased platelet count. In addition, Tajiri et al (23) observed that the significant increase in postoperative red blood cell and platelet counts may be maintained for 7.5-8 years following PSE if the degree of embolization approaches $70 \%$. Based upon previous results, the present study was conducted to investigate phased joint intervention with a small PSE area of $30-40 \%$ and a total embolization area of $60-80 \%$, which ensured efficacy and minimized the incidence of complications.

In the current study, phased joint intervention led to the immediate cessation of bleeding in 53 patients with an emergency hemostasis rate of $100 \%$, and led to the disappearance or significant relief of varices. A previous study reported that portal hemodynamics were improved and the number of WBCs and platelets were increased significantly following the intervention (16). The optimum range of splenic artery embolization was determined to be $60-80 \%$, as it did not result in

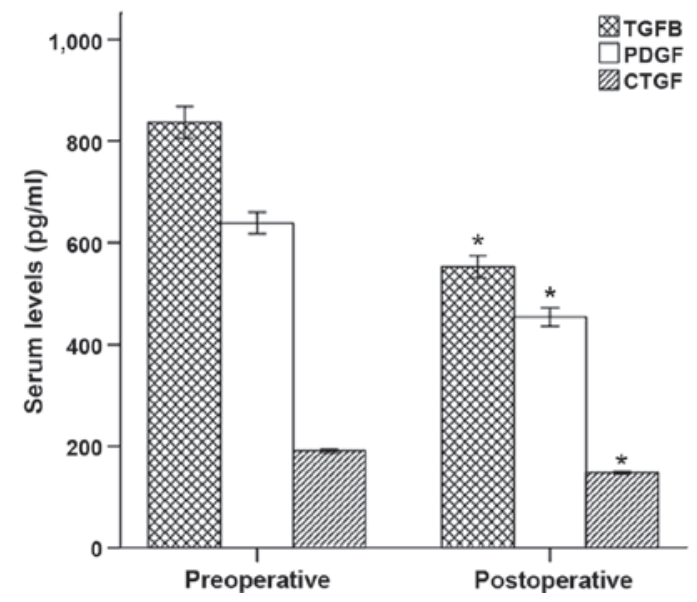

Figure 4. TGF- $\beta 1$, CTGF and PDGF concentrations in the peripheral blood of the preoperative and postoperative groups, determined by ELISA. TGF- $\beta 1$, CTGF and PDGF concentrations in the peripheral blood following intervention were significantly reduced compared with the preoperative parameters (TGF- $\beta 1,552.89 \pm 69.49$ vs. $837.19 \pm 99.14$; CTGF, $147.68 \pm 7.62$ vs. $190.94 \pm 9.66$; and PDGF, $454.30 \pm 57.74$ vs. $638.74 \pm 66.68)$. ${ }^{*} \mathrm{P}<0.001$ vs. the preoperative group. A, preoperative group; $\mathrm{B}$, postoperative group; TGF, transforming growth factor; CTGF, connective tissue growth factor; PDGF, platelet-derived growth factor.

severe adverse reactions. The majority of patients experienced low/medium fever for a short duration.

The present study consolidated upon the recent observations regarding the efficacy of joint interventional embolization. However, the cellular and molecular biological mechanisms related to improvement of liver function have yet to be elucidated.

The principal feature of hepatic fibrosis is hypothesized to be the activation of HSCs into myofibroblasts in the area of tissue inflammation and necrosis (24). HSCs are able to activate, synthesize and secrete ECM components (25). HSCs of the liver sinusoid have contractile activity similar to that of smooth muscle cells, and have an important role in the regulation of hepatic blood circulation $(26,27)$. The myofibroblast effect of activated HSCs is one of the most important features of portal hypertension (28). We envisage that phased joint intervention improves liver hemodynamics, thereby affecting the biological behavior of HSCs, including their proliferation and expression of cytokines and ECM components. The present study further investigated the effect of Rho-ROCK expression on phased joint interventional embolization, and explored the possible mechanisms of the effect of embolization on liver hemodynamics.

Rho small $\mathrm{G}$ proteins are associated with cell adhesion and cytoskeletal proteins, and belong to the small $\mathrm{G}$ protein superfamily, serving an important role in force transduction. Critical molecules of the Rho-ROCK signaling pathway include Rho, ROCK and myosin phosphatase target subunit 1 (MYPT1). ROCK receives activation signals from Rho and is phosphorylated at multiple amino acid sites for activation (29). Activated ROCK is associated with a number of diseases such as organ fibrosis through multiple cascade reactions that cause biological effects such as cell contraction, migration, adhesion, growth and division, production of stress fibers, movement of smooth muscles, and collagen synthesis $(30,31)$. 
The specific ROCK inhibitor Y-27632 is able to inhibit HSC activation, thus preventing rat liver fibrosis $(32,33)$. Y-27632 is also able to inhibit endothelin-induced HSC contraction and formation of portal hypertension (34).

The current study determined that mRNA expression levels of Rho, ROCK1 and ROCK2 were higher compared with the normal control group prior to and after phased joint embolization, indicating that the Rho-ROCK pathway is associated with the development of cirrhosis. mRNA expression levels of Rho, ROCK1 and ROCK2 following embolization were lower, compared with the preoperative levels. Protein expression levels were consistent with the mRNA expression levels. Postoperative pMYPT1 protein expression levels were significantly reduced, whereas tMYPT1 protein expression levels were not significantly affected. This indicates that phased joint embolization lowers Rho and ROCK expression levels and affects their activation.

TGF- $\beta 1$ is a critical cytokine in promoting liver fibrosis, and is able activate and transform HSCs into myofibroblasts, resulting in the production of ECM components and liver fibrosis (35). CTGF is a downstream effector of TGF- $\beta$, and mediates TGF- $\beta$ to promote cell proliferation and synthesize ECM $(36,37)$. Hahn et al (37) have indicated that Rho proteins and the entire cytoskeleton have an important role in the expression of CTGF and TGF- $\beta$ in fibroblasts. Sakata et al (14) used the Flexercell loading unit FX-2000 to prolong the cycle of artificially cultured human HSCs to decrease the TGF- $\beta$ concentration and mRNA expression levels. The negative mutant of transfected Rho factor is able to inhibit stretch-induced TGF- $\beta$ synthesis, which indicates that Rho is associated with stretch-induced TGF- $\beta$ synthesis in HSCs. Mechanical force is able to rapidly induce phosphorylation of the PDGF receptor, activation of integrin receptors, and stretching of activated cation channels and $G$ proteins. The aforementioned proteins may serve as mechanical sensors, thereby activating signal transmission pathways of growth factors (38).

The present study revealed that TGF- $\beta 1$, CTGF and PDGF concentrations in peripheral blood after phased joint intervention were significantly reduced in the postoperative group, compared with preoperative levels. Furthermore, the present findings indicated that phased joint embolization improves liver hemodynamics, reduces mRNA expression levels of Rho and ROCK, and inhibits the production of TGF- $\beta 1$, CTGF, PDGF and other cytokines, thus inhibiting the activation and proliferation of HSCs, thereby improving liver function and delaying the progression of cirrhosis.

The present preliminary study also confirmed that joint intervention is able to treat EVB and improve hypersplenism and liver function, effectively and safely without complications. Phased joint embolization improves liver hemodynamics, which may have a significant impact on the activation and proliferation of HSCs, as well as expression levels of ECM. This effect may be associated with the Rho-ROCK signaling pathway. However, the present study had an insufficient number of cases to be conclusive, with short-term follow-up and a limited assessment of the long-term outcomes subsequent to surgery. Therefore, further studies are required with a greater number of cases and a longer period of follow-up.

\section{References}

1. Comar KM and Sanyal AJ: Portal hypertensive bleeding. Gastroenterol Clin North Am 32: 1079-1105, 2003.

2. Augustin S, González A and Genescà J: Acute esophageal variceal bleeding: Current strategies and new perspectives. World J Hepatol 2: 261-274, 2010.

3. Kovalak M, Lake J, Mattek N, Eisen G, Lieberman D and Zaman A: Endoscopic screening for varices in cirrhotic patients: Data from a national endoscopic database. Gastrointest Endosc 65: 82-88, 2007.

4. Groszmann RJ, Garcia-Tsao G, Bosch J, Grace ND, Burroughs AK, Planas R, Escorsell A, Garcia-Pagan JC, Patch D, Matloff DS, et al: Beta-blockers to prevent gastroesophageal varices in patients with cirrhosis. N Engl J Med 353: 2254-2261, 2005.

5. Merli M, Nicolini G, Angeloni S, Rinaldi V, De Santis A, Merkel C, Attili AF and Riggio O: Incidence and natural history of small esophageal varices in cirrhotic patients. J Hepatol 38: 266-272, 2003.

6. D'Amico G, Pagliaro L and Bosch J: Pharmacological treatment of portal hypertension: An evidence-based approach. Semin Liver Dis 19: 475-505, 1999.

7. Bosch J and García-Pagán JC: Prevention of variceal rebleeding. Lancet 361: 952-954, 2003.

8. de Franchis R: Evolving consensus in portal hypertension. Report of the Baveno IV consensus workshop on methodology of diagnosis and therapy in portal hypertension. J Hepatol 43: 167-176, 2005.

9. Lunderquist A and Vang J: Sclerosing injection of esophageal varices through transhepatic selective catheterization of the gastric coronary vein. A preliminary report. Acta Radiol Diagn (Stockh) 15: 546-550, 1974.

10. Benner KG, Keeffe EB, Keller FS and Rösch J: Clinical outcome after percutaneous transhepatic obliteration of esophageal varices. Gastroenterology 85: 146-153, 1983.

11. Chikamori F, Kuniyoshi N, Shibuya S and Takase Y: Correlation between endoscopic and angiographic findings in patients with esophageal and isolated gastric varices. Dig Surg 18: 176-181, 2001.

12. L'Herminé C, Chastanet P, Delemazure O, Bonnière PL, Durieu JP and Paris JC: Percutaneous transhepatic embolization of gastroesophageal varices: Results in 400 patients. AJR Am J Roentgenol 152: 755-760, 1989.

13. Koconis KG, Singh H and Soares G: Partial splenic embolization in the treatment of patients with portal hypertension: A review of the english language literature. J Vasc Interv Radiol 18: 463-481, 2007.

14. Sakata R, Ueno T, Nakamura T, Ueno H and Sata M: Mechanical stretch induces TGF-beta synthesis in hepatic stellate cells. Eur J Clin Invest 34: 129-136, 2004.

15. Okada Y, Tsuzuki Y, Hokari R, Miyazaki J, Matsuzaki K, Mataki N, Komoto S, Watanabe C, Kawaguchi A, Nagao S, et al: Pressure loading and ethanol exposure differentially modulate rat hepatic stellate cell activation. J Cell Physiol 215: 472-480, 2008.

16. Wang Y, Dong J, Meng W, Ma J, Wang N, Wei J and Shi M: Effects of phased joint intervention on IL-35 and IL-17 expression levels in patients with portal hypertension. Int J Mol Med 33: 1131-1139, 2014.

17. Tangkijvanich P, Tam SP and Yee HF Jr.: Wound-induced migration of rat hepatic stellate cells is modulated by endothelin-1 through rho-kinase-mediated alterations in the acto-myosin cytoskeleton. Hepatology 33: 74-80, 2001.

18. Livak KJ and Schmittgen TD: Analysis of relative gene expression data using real-time quantitative PCR and the 2(-Delta Delta C(T)) Method. Methods 25: 402-408, 2001.

19. Sun A, Shi YJ, Xu ZD, Tian XG, Hu JH, Wang GC and Zhang CQ: MDCT angiography to evaluate the therapeutic effect of PTVE for esophageal varices. World J Gastroenterol 19: 1563-1571, 2013.

20. Hadduck TA and McWilliams JP: Partial splenic artery embolization in cirrhotic patients. World J Radiol 6: 160-168, 2014.

21. Sangro B, Bilbao I, Herrero I, Corella C, Longo J, Beloqui O, Ruiz J, Zozaya JM, Quiroga J and Prieto J: Partial splenic embolization for the treatment of hypersplenism in cirrhosis. Hepatology 18: 309-314, 1993.

22. Pålsson B, Hallén M, Forsberg AM and Alwmark A: Partial splenic embolization: Long-term outcome. Langenbecks Arch Surg 387: 421-426, 2003. 
23. Tajiri T, Onda M, Yoshida H, Mamada Y, Taniai $N$ and Kumazaki T: Long-term hematological and biochemical effects of partial splenic embolization in hepatic cirrhosis. Hepatogastroenterology 49: 1445-1448, 2002.

24. Wang Y, Gao J, Zhang D, Zhang J, Ma J and Jiang H: New insights into the antifibrotic effects of sorafenib on hepatic stellate cells and liver fibrosis. J Hepatol 53: 132-144, 2010.

25. Parsons CJ, Bradford BU, Pan CQ, Cheung E, Schauer M, Knorr A, Krebs B, Kraft S, Zahn S, Brocks B, et al: Antifibrotic effects of a tissue inhibitor of metalloproteinase-1 antibody on established liver fibrosis in rats. Hepatology 40: 1106-1115, 2004.

26. Pinzani M: Hepatic stellate (ITO) cells: Expanding roles for a liver-specific pericyte. J Hepatol 22: 700-706, 1995

27. Pinzani M, Failli P, Ruocco C, Casini A, Milani S, Baldi E, Giotti A and Gentilini P: Fat-storing cells as liver-specific pericytes. Spatial dynamics of agonist-stimulated intracellular calcium transients. J Clin Invest 90: 642-646, 1992.

28. Thoen LF, Guimarães EL, Dollé L, Mannaerts I, Najimi M, Sokal E and van Grunsven LA: A role for autophagy during hepatic stellate cell activation. J Hepatol 55: 1353-1360, 2011

29. Brown JH, Del Re DP and Sussman MA: The Rac and Rho hall of fame: A decade of hypertrophic signaling hits. Circ Res 98: 730-742, 2006

30. Hennenberg M,BieckerE, Trebicka J,JochemK,Zhou Q,Schmidt M Jakobs KH, Sauerbruch T and Heller J: Defective RhoA/Rho-kinase signaling contributes to vascular hypocontractility and vasodilation in cirrhotic rats. Gastroenterology 130: 838-854, 2006.
31. Riento K, Guasch RM, Garg R, Jin B and Ridley AJ: RhoE binds to ROCK I and inhibits downstream signaling. Mol Cell Biol 23: 4219-4229, 2003.

32. Murata T, Arii S, Mori A and Imamura M: Therapeutic significance of $y-27632$, a Rho-kinase inhibitor, on the established liver fibrosis. J Surg Res 114: 64-71, 2003.

33. Klein S, Van Beuge MM, Granzow M, Beljaars L, Schierwagen R, Kilic S, Heidari I, Huss S, Sauerbruch T, Poelstra K and Trebicka J: HSC-specific inhibition of Rho-kinase reduces portal pressure in cirrhotic rats without major systemic effects. J Hepatol 57: 1220-1227, 2012.

34. Kawada N, Seki S, Kuroki T and Kaneda K: Rock inhibitor y-27632 attenuates stellate cell contraction and portal pressure increase induced by endothelin-1. Biochem Biophys Res Commun 266: 296-300, 1999.

35. Bissell DM, Roulot D and George J: Transforming growth factor beta and the liver. Hepatology 34: 859-867, 2001.

36. Boettner B and Van Aelst L: The role of Rho GTPases in disease development. Gene 286: 155-174, 2002.

37. Hahn A, Heusinger-Ribeiro J, Lanz T, Zenkel S and Goppelt-Struebe M: Induction of connective tissue growth factor by activation of heptahelical receptors. Modulation by Rho proteins and the actin cytoskeleton. J Biol Chem 275: 37429-37435, 2000.

38. Li C and Xu Q: Mechanical stress-initiated signal transductions in vascular smooth muscle cells. Cell Signal 12: 435-445, 2000. 